\title{
Momelotinib sensitizes glioblastoma cells to temozolomide by enhancement of autophagy via JAK2/STAT3 inhibition
}

\author{
TIE LIU, ANQI LI, YULUN XU and YU XIN \\ Department of Neurosurgery, Beijing Tiantan Hospital, Capital Medical University, \\ Dongcheng, Beijing 100050, P.R. China
}

Received July 26, 2018; Accepted January 8, 2019

DOI: $10.3892 /$ or.2019.6970

\begin{abstract}
Temozolomide (TMZ) is a widely used chemotherapeutic agent for glioblastoma multiforme (GBM). However, chemoresistance to TMZ is still a major obstacle for GBM patients. An abundance of candidates has been reported to improve the chemotherapeutic sensitization of TMZ. In the present study, it was demonstrated that momelotinib (MTB) enhanced the sensitivity of glioma cells to TMZ in vitro, as evidenced by a noticeable decrease in cell growth and a significant increase in apoptosis and autophagy following treatment with the combination of TMZ and MTB compared to TMZ alone. Mechanistically, MTB and TMZ combination treatment reduced $\mathrm{U} 251$ cell growth by activating both apoptosis and autophagy pathways. MTB potentiated TMZ to inhibit the phosphorylation of JAK2 and STAT3 in U251 cells, resulting in the inactivation of JAK2/STAT3 signaling pathways. Moreover, we investigated the effect of MTB in xenograft tumor model mice. MTB and TMZ combination reduced tumor weight, decreased the expression of $\mathrm{Ki}-67$, P62, p-STAT3 and p-JAK2, while increased the ratio of LC3-II/I and the expression of caspase-3 and Beclin1 in vivo. Importantly, this combination was well tolerated, and caused significant tumor growth inhibition in the GBM xenografts. In summary, the present study provides pharmacological evidence that MTB has potential value in the treatment of GBM.
\end{abstract}

Correspondence to: $\mathrm{Dr} \mathrm{Yu}$ Xin, Department of Neurosurgery, Beijing Tiantan Hospital, Capital Medical University, 6 Tiantan Xili, Dongcheng, Beijing 100050, P.R. China

E-mail: yuxin_new@163.com

Abbreviations: MTB, momelotinib; TMZ, temozolomide; GBM, glioblastoma multiforme; TUNEL, terminal deoxynucleotidyl transferase-mediated dUTP-biotin nick end labeling; p-STAT3, Tyr705-phosphorylated STAT3; DMSO, dimethyl sulfoxide; JAK2, janus kinase 2

Key words: momelotinib, glioblastoma, temozolomide, chemosensitivity

\section{Introduction}

Glioblastoma (GBM) is one of the most prevalent malignant cancers and is the most lethal primary intrinsic brain tumor (1). Currently, even following standard treatment, namely, surgical excision followed by radiotherapy alone or adjuvant chemotherapy, patients exhibit a poor prognosis, with long-term survival rates of $<5 \%(2,3)$. The clinical course of GBM is very rapid and fatal. According to a previous investigation concerning glioblastoma survival in the United States, the median survival following standard treatment and adjuvant chemotherapy with temozolomide (TMZ) was only 15 months (4). TMZ is an alkylating agent (current front-line GBM therapeutic drug) administered orally which causes DNA damage and induces cell death. TMZ exhibits anti-glioma activity when used alone or in combination with other agents (5). TMZ has been approved for the clinical treatment of patients with newly diagnosed malignant glioma and recurrent malignant glioma $(3,6)$. Athanassiou et al revealed the therapeutic mechanisms of TMZ. The authors demonstrated that TMZ can efficiently inhibit the proliferation and induce the apoptosis of glioma cells (3). However, GBM cells usually achieve resistance to TMZ which alleviates the cell toxicity of TMZ. The occurrence of chemoresistance to $\mathrm{TMZ}$ is the main cause of the poor prognosis of GBM patients. Improving the sensitivity of GBM cells to TMZ has become a promising strategy for GBM treatment.

STAT3 is a transcription factor and one of the major intracellular signaling proteins. STAT3 has been found to be upregulated in GBM (7). STAT3 can be activated by phosphorylation of the STAT3 tyrosine 705 residue, which is stimulated by JAK2 activation under any of a variety of cytokines including IL-6 and IL-11 as well as growth factors such as EGF, TGF- $\alpha$, PDGF and HGF (8). Upon activation, Tyr705-phosphorylated STAT3 (p-STAT3) translocates from the cytoplasm to the nucleus, thus regulating the transcription of downstream target genes by binding to the promoter of these genes, including Bcl-2, Bcl-xL and vascular endothelial growth factor (VEGF) (7). Constitutive activation of STAT3 has been confirmed to be positively correlated with the grade of glioma (9). JAK2/STAT3 inhibition leads to apoptosis (10) and autophagy (11). JAK2/STAT3 is a well-known target of GBM treatment $(12,13)$. 
Momelotinib (MTB; also named CYT387). is a smallmolecule, adenosine triphosphate (ATP)-competitive inhibitor of janus kinase (JAK)-1/2 (14,15). MTB has been evaluated in phase 3 myelofibrosis clinical trials (NCT01969838 and NCT02101268) (16). MTB was reported to inhibit the growth of pancreatic ductal adenocarcinoma (PDAC) (17) and ovarian carcinoma cells (18). In addition, a previous study showed that MTB and dasatinib synergistically inhibited renal cell carcinoma (19). Similarly, MTB was reported to enhance the antitumor activity of cetuximab against non-small cell lung cancer (20). These results indicated that MTB has anticancer potential. In the present study, we aimed to investigate whether MTB sensitizes GBM cells to TMZ and the underlying mechanisms.

\section{Materials and methods}

Cell culture and reagents. The human GBM cell lines U373-MG-ATCC, U251, SHG-44 and LN229 (CRL-2611) were purchased from the American Type Culture Collection (ATCC; Manassas, VA, USA). The human GBM cell lines U251, SHG-44 and LN229 were cultured in Dulbecco's modified essential medium (DMEM; Gibco; Thermo Fisher Scientific, Inc., Waltham, MA, USA) containing 10\% fetal bovine serum (FBS; Gibco; Thermo Fisher Scientific, Inc.), $100 \mathrm{U} / \mathrm{ml}$ penicillin + streptomycin (Gibco; Thermo Fisher Scientific, Inc.). U373-MG-ATCC cells were maintained in complete EMEM medium (Gibco; Thermo Fisher Scientific, Inc.) containing $10 \% \mathrm{FBS}$ and $100 \mathrm{U} / \mathrm{ml}$ penicillin + streptomycin (Gibco; Thermo Fisher Scientific, Inc.). All cell cultures were maintained at $37^{\circ} \mathrm{C}$ in a $5 \% \mathrm{CO}_{2}$ incubator. MTB was purchased from Selleckchem (S2219) and TMZ was purchased from Sigma-Aldrich (Merck KGaA, Darmstadt, Germany). Stock solutions were made in dimethyl sulfoxide (DMSO; Sigma-Aldrich; Merck KGaA) and subsequently diluted in culture medium for use. Working concentrations were freshly prepared daily by diluting the stock with phosphate-buffered saline (PBS).

Cell Counting Kit-8 cell viability assay. Cell Counting Kit-8 (CCK-8; Beyotime Institute of Biotechnology, Shanghai, China) assay was performed to test cell toxicity of MTB and proliferation of U373-MG-ATCC, U251, SHG-44 and LN229 cells in this study. For toxicity test, we chose astrocytes as a control. U-251MG cells treated with MTB $(0,0.1,0.025,0.05$, $0.1,0.25,0.5,1.0,2.5,5,10,25,50,100$ and $200 \mu \mathrm{M})$ for $48 \mathrm{~h}$, were collected to detect the optical density (OD) 490. For proliferation determination, $\mathrm{U}-251 \mathrm{MG}$ cells were pretreated with NC, $2.5 \mu \mathrm{M}$ MTB, $100 \mu \mathrm{M} \mathrm{TMZ}$ and MTB + TMZ for $24 \mathrm{~h}$, and then cells were trypsinized and seeded into 96-well plates at a density of $4 \times 10^{3}$ cells in $200 \mu \mathrm{l}$ of complete medium per well. Next, the 96 -well plates were placed at $37^{\circ} \mathrm{C}$ in a $5 \%$ $\mathrm{CO}_{2}$ incubator. CCK-8 assay was performed according to the manufacturer's instructions provided in the CCK- 8 kit for the next continuous 4 days. The value of $450 \mathrm{~nm}$ was measured by ELISA. This assay was repeated for 3 times.

Treatments of GBM cells. U-251MG cells were divided into 4 groups and treated with NC (Ctrl), $2.5 \mu \mathrm{M}$ MTB (MTB), $100 \mu \mathrm{M}$ TMZ (TMZ) and combination treatment $(2.5 \mu \mathrm{M}$
$\mathrm{MTB}+100 \mu \mathrm{M} \mathrm{TMZ}$ ), separately. TMZ at a concentration of $100 \mu \mathrm{M}$ was used as previously reported (21).

Hoechst 33342 staining. U-251MG cells were treated as described above for $48 \mathrm{~h}$. Briefly, cells in each group were detached from the culture dish and resuspended in DMEM containing $2 \%$ FBS at a density of $1 \times 10^{6}$ cells $/ \mathrm{ml}$. Then, the cells were incubated with Hoechst 33342 staining solution (Sigma-Aldrich; Merck KGaA) for $60 \mathrm{~min}$ at $37^{\circ} \mathrm{C}$ according to the manufacturer's instructions. Finally, the cells were observed under a fluorescence microscope (scale bar, $50 \mu \mathrm{m}$ ). Apoptotic cell death was determined by counting the number of cells with condensed nuclei in 6 randomly selected areas.

Western blot analysis. U-251MG cells were treated with MTB $(2.5 \mu \mathrm{M})$ or TMZ $(100 \mu \mathrm{M})$ for $48 \mathrm{~h}$. Proteins were extracted with RIPA lysis buffer adding cocktail tablets (Sigma Aldrich; Merck KGaA) and phenylmethanesulfonyl fluoride (PMSF; Sigma-Aldrich; Merck KGaA). An equivalent amount of protein $(40 \mu \mathrm{g})$ was resolved on SDS-PAGE (6-12\%) gel, transferred onto polyvinylidene fluoride (PVDF) membranes (EMD Millipore, Billerica, MA, USA) and probed with specific primary antibodies. The primary antibodies against cleaved caspase-9, Ki-67, PCNA, cleaved caspase-3, Beclin1, P62, LC3, phosphorylated (Tyr705) STAT3 (p-STAT3), STAT3, phosphorylated (Tyr1007/1008) JAK2 (p-JAK2), JAK2, were obtained from the Cell Signaling Technology, Inc. (Danvers, MA, USA) (Table I). After $1 \mathrm{~h}$ of incubation with the appropriate horseradish peroxidase (HRP)-conjugated secondary antibody (Santa Cruz Biotechnology, Inc., Dallas, TX, USA). Finally, the blots were visualized by ECL and detected using a ChemiDoc XRS imaging system (Bio-Rad Laboratories, Hercules, CA, USA). Protein bands were normalized with GAPDH as an internal control.

Immunofluorescence staining. Immunofluorescence staining was performed to confirm the localization of p-STAT3 in U-251MG cells. U-251MG cells were treated and grouped as previously described in the manuscript. Then, the cells were collected and washed with PBS twice and fixed with $4 \%$ paraformaldehyde for $20 \mathrm{~min}$ at room temperature. Next, the samples were permeabilized with $0.4 \%$ Triton X-100 (Sigma-Aldrich; Merck KGaA) for 10 min, washed with PBS and then blocked with $2 \%$ bovine serum albumin (BSA; Beijing Solarbio Science and Technology Co., Ltd., Beijing, China) in PBS for $1 \mathrm{~h}$ at $37^{\circ} \mathrm{C}$. After this, the samples were incubated with anti-p-STAT3 in 1\% BSA (Beijing Solarbio Science and Technology Co., Ltd.) in PBS overnight at $4{ }^{\circ} \mathrm{C}$. The samples were subsequently washed with PBS and incubated with fluorescein-conjugated secondary antibody in $1 \%$ BSA (Beijing Solarbio Science and Technology Co., Ltd.) in PBS for $1 \mathrm{~h}$. Finally, the samples were stained with DAPI $(1 \mu \mathrm{g} / \mathrm{ml})$ for $10 \mathrm{~min}$ to stain the cell nuclei. The samples were then visualized a confocal laser scanning microscope at $\mathrm{x} 400$ magnification (Leica Microsystems, GmbH, Wetzlar, Germany).

Cell cycle assay. U-251MG cells were seeded in 6-well cell culture plates. After $48 \mathrm{~h}$ of treatment with TMZ with or without MTB, the cells were analyzed by flow cytometry (BD FACSCanto II; BD Biosciences, San Diego, CA, USA) 
Table I. Primary and secondary antibody information.

\begin{tabular}{llcr}
\hline Antibodies & \multicolumn{1}{c}{ Company } & Catalog no. & Dilution \\
\hline Primary cleaved caspase-9 & Cell Signaling Technology (CST), Inc., & 52,873 & $1: 2,000$ \\
& Danvers, MA, USA & & $1: 2,000$ \\
Cleaved caspase-3 & CST & 9,662 & $1: 2,000$ \\
Ki-67 & Abcam, Cambridge, UK & ab16667 & $1: 2,000$ \\
PCNA & CST & 13,110 & $1: 1,000$ \\
Beclin1 & CST & 3,495 & $1: 1,000$ \\
p62 & CST & 8,025 & $1: 1,000$ \\
LC3 & CST & 4,108 & $1: 2,000$ \\
STAT3 & CST & 9,139 & $1: 2,000$ \\
p-STAT3 (Tyr705) & CST & 9,145 & $1: 1,000$ \\
p-JAK2 & CST & 3,771 & $1: 1,000$ \\
Secondary (Tyr1007/1008) & & & $1: 10,000$ \\
JAK2 & CST & 3,230 & \\
m-IgGк BP-HRP & Santa Cruz Biotechnology, Inc. & sc-516102 \\
& Dallas, TX, USA & & \\
\hline
\end{tabular}

using CycleTEST ${ }^{\text {тм }}$ Plus DNA Reagent kit (BD Biosciences) according to the manufacturer's recommended protocol. The obtained results were analyzed using ModFit LT (version 4.0; Verity Software House, Topsham, ME, USA).

Xenograft tumor model. For testing of the efficacy of MTB combined with TMZ in vivo, orthotopic xenograft models were established. Twenty 6-week-old male BALB/c-nu/nu mice weighing 16-18 $\mathrm{g}$ were bred in aseptic cages, which were kept in an isolator unit with filtered air. The mice had access to water and food ad libitum. U251 cell suspension $(0.1 \mathrm{ml})$ [2-5x $10^{7} / \mathrm{ml}$ Hank's balanced salt solution (HBSS), Gibco; Thermo Fisher Scientific, Inc.] was stereotypically injected into the left striata of mice. After the tumor was established, the mice were randomized into the following groups: Ctrl; MTB (50 mg/kg/day body weight) (19); TMZ (20 mg/kg/day body weight); MTB + TMZ (50 mg/kg/day MTB + $20 \mathrm{mg} / \mathrm{kg} /$ day TMZ). All mice were administered the drug or saline daily by oral gavage 1 day after tumor cell injection (18). All animals were sacrificed at 30 days and tumors were collected. Tumor weight was measured. All animal protocols were approved by the Beijing Tiantan Hospital and Capital Medical university laws governing animal care.

TUNEL. Terminal deoxynucleotidyl transferase-mediated dUTP-biotin nick-end labeling (TUNEL) staining was performed to detect apoptosis according to the manufacturer's instructions (TUNEL Apoptosis Detection Kit; Upstate Biotechnology, Inc., Lake Placid, NY, USA). TUNEL-positive cells were quantified by counting brown staining within the nucleus of apoptotic cells. The extent of apoptosis was calculated with the Motic Image software (version 1.2; Micro Optical Group Co., Haimen, China) and expressed as a number of TUNEL-positive cells from the same field under an Olympus BX60 microscope and Spot camera (Olympus Optical Co., Tokyo, Japan; magnification, x400).
Immunohistochemistry. Tumors were cut into $2-\mu \mathrm{m}$ sections and paraffin-embedded tumor sections were constructed. Sections were deparaffinized and blocked with $3 \% \mathrm{H}_{2} \mathrm{O}_{2}$ for $10 \mathrm{~min}$ at room temperature followed by incubation with anti-Ki-67 and anti-cleaved caspase- 3 at $4^{\circ} \mathrm{C}$ overnight. Slides were rinsed with PBS and incubated at room temperature for 30 min with biotinylated goat anti-mouse immunglobulin G secondary antibody (Dako, Glostrup, Denmark). After washing in Tris-hydrochloric acid buffer (TBS), the slides were incubated with streptavidin-peroxidase reagent (Dako) and treated with 3,3'-diaminobenzidine (DAB; Sigma Aldrich; Merck $\mathrm{KGaA}$ ) for $5 \mathrm{~min}$. The slides were counterstained and dehydrated and the final percentage of positive cells was calculated with the Motic Image software (version 1.2; Micro Optical Group Co.)

Autophagy detection. Autophagy was assessed by western blot analysis and immunofluorescence staining for LC3. Detailed methods were shown in paragraph Western blot analysis and Immunofluorescence staining. All stained specimens were observed under a confocal laser scanning microscope (Leica Microsystems, GmbH). For the quantitative analysis, LC3 positive puncta were analyzed by ImageJ (version 1.50b; National Institutes of Health, Bethesda, ME, USA; http://imagej.nih. gov/ij/).

Statistical analysis. Statistically significant differences between the control and other groups were evaluated by means of analysis of variance (ANOVA). For comparisons among $>2$ groups, the one-way ANOVA followed by post hoc Tukey's test was applied. For the CCK-8 assay, the one-way ANOVA with Dunnett's post hoc test was used. Data are illustrated in bar graphs, values are presented as mean \pm SEM from at least 3 independent experiments and post hoc test. Asterisks/pound symbols denote statistical significance in the figures. Statistically significant differences in Kaplan-Meier 


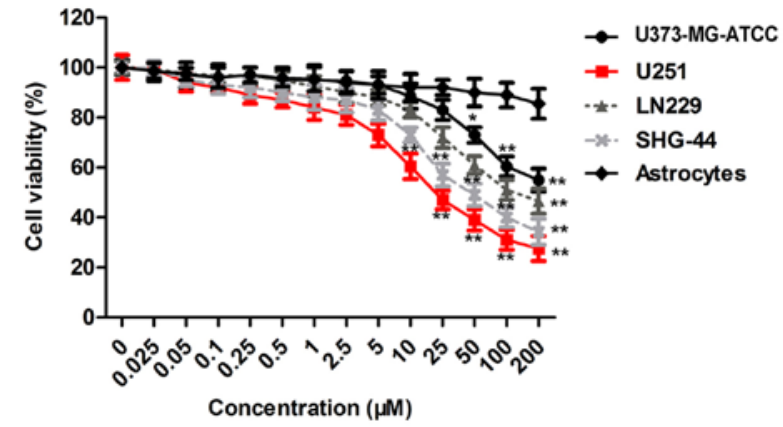

Figure 1. MTB inhibits the cell viability of 4 human GBM cell lines. MTB at various concentrations $(0-200 \mu \mathrm{M})$ was used to treat GBM cell lines U373-MG-ATCC, U251, SHG-44 and LN229 and astrocytes. "P<0.05, ${ }^{* *} \mathrm{P}<0.01$, compared with the astrocyte group. MTB, momelotinib; GBM, glioblastoma; U251, U251-MG.

survival were determined by a log-rank test using GraphPad Prism. Statistical significance was considered if $\mathrm{P}<0.05$ in any analysis. All the data were analyzed using GraphPad Prism 6 (GraphPad Software, Inc., San Diego, CA, USA).

\section{Results}

$M T B$ inhibits the cell viability of 4 human GBM cell lines. To clarify the influence of MTB on GBM cells, we conducted a CCK-8 assay using U373-MG-ATCC, U251, SHG-44 and LN229 cells and astrocytes treated with MTB at concentrations of 0 to $200 \mu \mathrm{M}$ for $24 \mathrm{~h}$, respectively. The result (Fig. 1) showed that MTB exerted an inhibitory effect on the viability of GBM cells in a dose-dependent manner $(\mathrm{P}<0.01$; compared to the astrocyte group). Particularly, U251 cells showed more sensitivity to MTB than the other cell lines. The concentration of MTB that inhibited cell viability was $2.5 \mu \mathrm{M}$ in U251 cells. In addition, treatment with MTB at $2.5 \mu \mathrm{M}$ did not induce significant cytotoxicity of U251 cells. These data suggest that MTB is a potential anticancer agent against GBM cells, particularly U251 cells. Thus, $2.5 \mu \mathrm{M}$ of MTB and U251 cells were used for subsequent experiments.

$M T B$ enhances the proliferation-inhibiting and apoptosispromoting effect of TMZ. A concentration of $100 \mu \mathrm{M}$ TMZ was used as previously described (21). As shown in Fig. 2A,
A
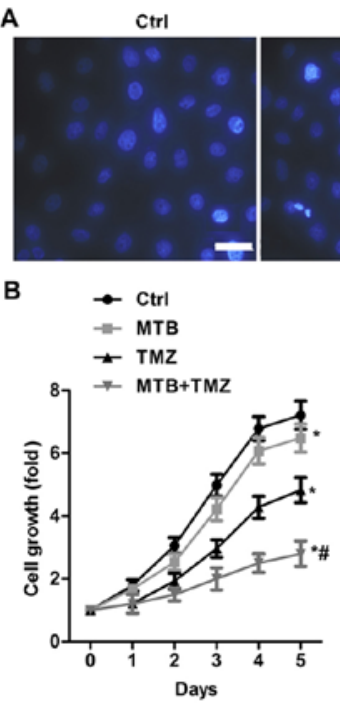

MTB

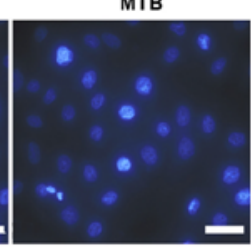

C

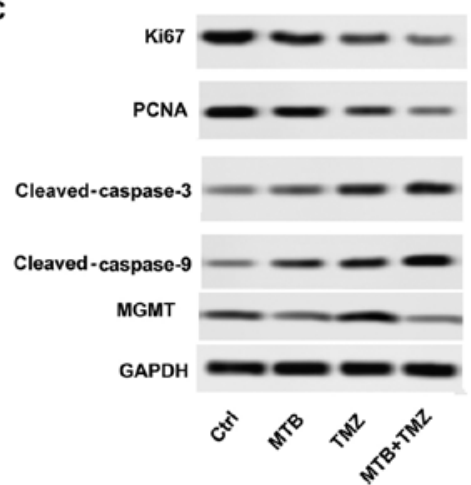

TMZ
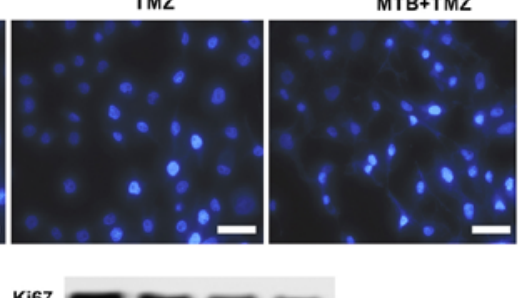
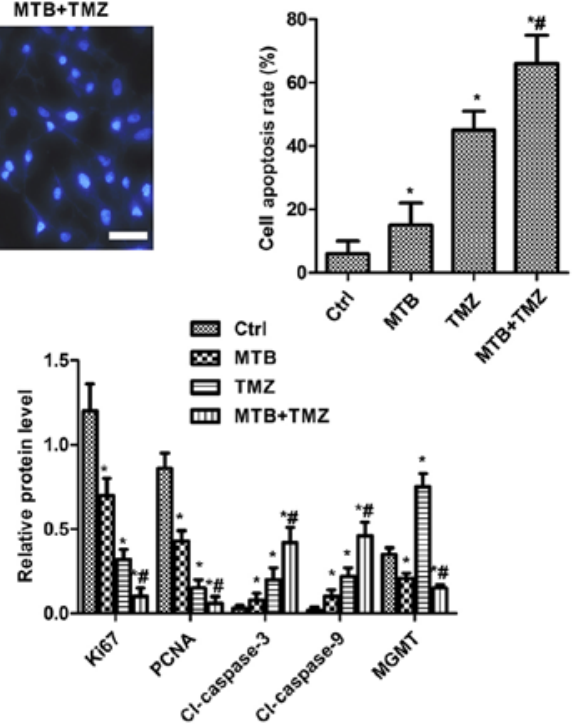

D

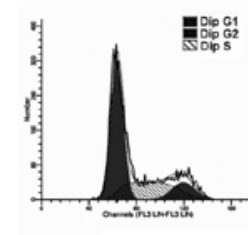

CtrI

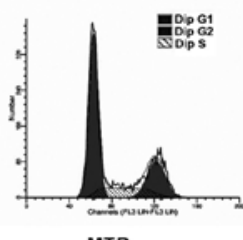

MTB

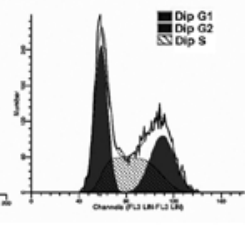

TMZ
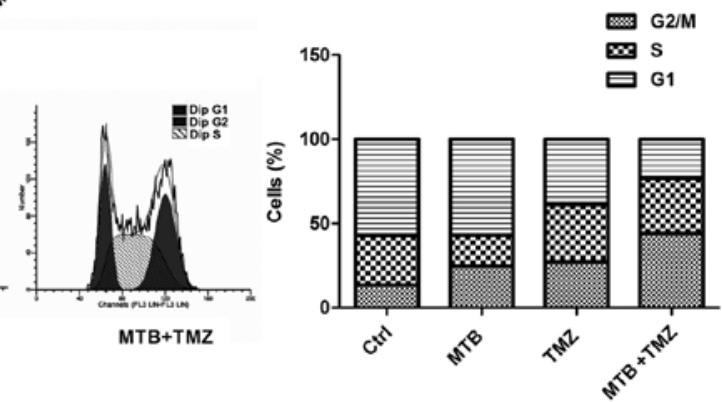

Figure 2. MTB enhances the proliferation-inhibiting and apoptosis-promoting effect of TMZ in GBM cells. The U251 cell line was selected for subsequent experiments. U251 cells were treated with DMSO (control), $2.5 \mu \mathrm{M} \mathrm{MTB,} 100 \mu \mathrm{M}$ TMZ, or $2.5 \mu \mathrm{M}$ MTB combined with $100 \mu \mathrm{M}$ TMZ (MTB + TMZ). (A) Apoptosis of cells under different treatments was detected by Hoechst 33258 staining (left panels) Scale bar, $50 \mu \mathrm{m}$. The representative histogram showing the results of the apoptosis rate of U251 cells (right panel). (B) Cells were cultured in 96-well plates and then treated with solvent only (control; Ctrl) or $100 \mu \mathrm{M}$ TMZ with $2.5 \mu \mathrm{M}$ MTB for $48 \mathrm{~h}$. OD $_{490}$ of U251 cells treated with MTB and TMZ were determined by CCK-8 assay. (C) The protein expression of cells under different treatments was detected by western blot analysis. GAPDH was used as the loading control (left image). The representative column diagrams showing results of relative protein expression (right histogram). (D) Cell cycle distribution was detected by FACS cell cycle analysis (left panels) and the representative histogram showing cell cycle distribution (right histogram). ${ }^{*} \mathrm{P}<0.05$, compared with the Ctrl; ${ }^{\prime} \mathrm{P}<0.05$, compared with TMZ. Data are represented as mean \pm SEM for 3 replicates. MTB, momelotinib; TMZ, temozolomide; GBM, glioblastoma; PCNA, proliferating cell nuclear antigen; MGMT, $O$-methylguanine-DNA methyltransferase; Ctrl, control. 


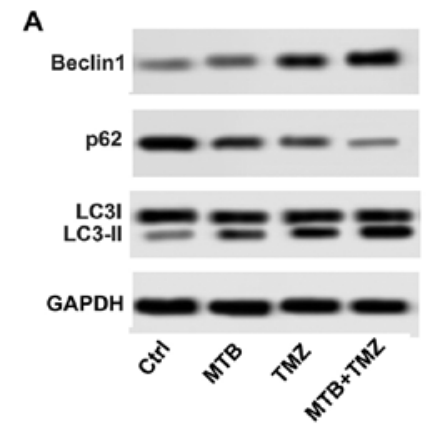

B

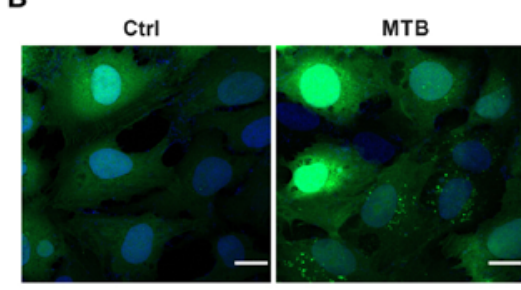

C

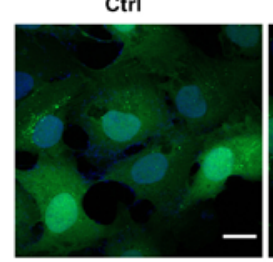

TMZ

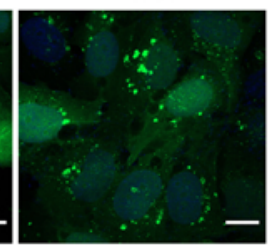

TMZ+3-MA

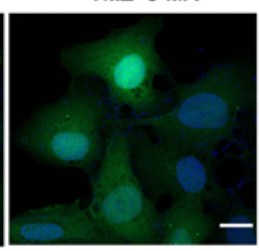

$T M Z+3-M A+M T B$

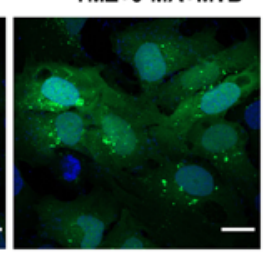

Ctrl

MTB

曰 TMZ

血 MTB+TMZ

Figure 3. MTB enhances the autophagy-promoting effect of TMZ in GBM cells. 3-MA was used to verify the autophagy-promoting effect of TMZ. (A) U251 cells were treated with DMSO (Ctrl), $2.5 \mu \mathrm{M}$ MTB, $100 \mu \mathrm{M} \mathrm{TMZ}$, or $2.5 \mu \mathrm{M}$ MTB combined with $100 \mu \mathrm{M} \mathrm{TMZ} \mathrm{(MTB+TMZ).} \mathrm{Relative} \mathrm{protein} \mathrm{levels} \mathrm{of}$ Beclin1, LC3 I/II and p62 were detected by western blot analysis. GAPDH was used as loading control. The quantification of relative protein expression was performed by ImageJ software. (B) Expression of LC3 was visualized with fluorescence microscopy after immunofluorescence staining with the LC 3 antibody (green); scale bar, $20 \mu \mathrm{m}$. The number of $\mathrm{LC}^{+}$puncta/cell was compared between the different groups. " $\mathrm{P}<0.05$, compared with $\mathrm{Ctrl}$; ${ }^{\#} \mathrm{P}<0.05$, compared with TMZ. (C) U251 cells were treated with DMSO (Ctrl), $100 \mu \mathrm{M} \mathrm{TMZ,} 100 \mu \mathrm{M} \mathrm{TMZ}+10 \mathrm{mM} 3-\mathrm{MA}$ (TMZ+3-MA) and $2.5 \mu \mathrm{M} \mathrm{MTB}+100 \mu \mathrm{M} \mathrm{TMZ}+10 \mathrm{mM}$ 3-MA (TMZ + 3-MA + MTB). Expression of LC3 was visualized with fluorescence microscopy after immunofluorescence staining with LC3 antibody (green); scale bar, $20 \mu \mathrm{m}$. The number of $\mathrm{LC}^{+}$puncta/cell was compared between the different groups. ${ }^{*} \mathrm{P}<0.05$, compared with Ctrl; " $\mathrm{P}<0.05$, compared with TMZ; ${ }^{\star} \mathrm{P}<0.05$, compared with TMZ+3-MA. The results are presented as the mean of 3 independent experiments. MTB, momelotinib; TMZ, temozolomide; GBM, glioblastoma; 3-MA, 3-methyladenine; LC3, microtubule-associated protein 1 light chain 3; Ctrl, control.

both MTB and TMZ promoted the apoptosis of U251 cells compared with the control $(\mathrm{P}<0.05)$. As shown in Fig. 2B, both MTB and TMZ suppressed the growth of U-251MG cells compared with the control $(\mathrm{P}<0.05)$. However, the combination of MTB and TMZ enhanced the proliferation-inhibiting effect compared with either the MTB or TMZ group $(\mathrm{P}<0.05)$. In addition, the combination of MTB and TMZ enhanced the apoptosis-promoting effect compared to TMZ or MTB used alone at the same dose $(\mathrm{P}<0.05)$. The present results were verified by western blot analysis of Ki-67, PCNA, cleaved caspase-3 and cleaved caspase-9 (Fig. 2C). The relative expression of Ki-67 and PCNA were significantly reduced by MTB and TMZ, and the combination therapy $(\mathrm{P}<0.05)$, while the expression of cleaved caspase- 3 and cleaved caspase- 9 exhibited inverse results. We also assessed the effect of MTB on cell cycle distribution (Fig. 2D). MTB and TMZ combination significantly arrested the cell cycle at the $\mathrm{G} 2$ phase.

MTB enhances the autophagy-promoting effect of TMZ. To elucidate the role of MTB in autophagy, expression levels of
Beclin1, LC3-II, LC3-I and p62 were detected by western blot assay. As shown in Fig. 3A, both MTB and TMZ increased the level of Beclin1 and LC3-II and decreased the level of p62 compared to the control $(\mathrm{P}<0.05)$. Expectedly, the combination of MTB and TMZ enhanced the autophagy-promoting effect, evidenced by higher levels of Beclin1 and LC3-II/I ratio, as well as a lower level of P62 compared with the MTB and TMZ group $(\mathrm{P}<0.05)$. In addition, the combination of MTB and TMZ obviously increased the number of LC3-positive puncta per cell when compared to that when TMZ or MTB was used alone at the same dose (Fig. 3B, P<0.05). 3-Methyladenine (3-MA) was used to verifiy the autophagy-promoting effect of MTB. As shown in Fig. 3C, TMZ alone significantly increased the number of LC3-positive puncta, and 3-MA totally inhibited the autophagy induced by TMZ, while the suppression was reversed by MTB.

MTB inactivates the JAK2/STAT3 pathway. To elucidate the mechanism of MTB in autophagy induction, expression levels of JAK2 and STAT3 were determined by western blot analysis. 


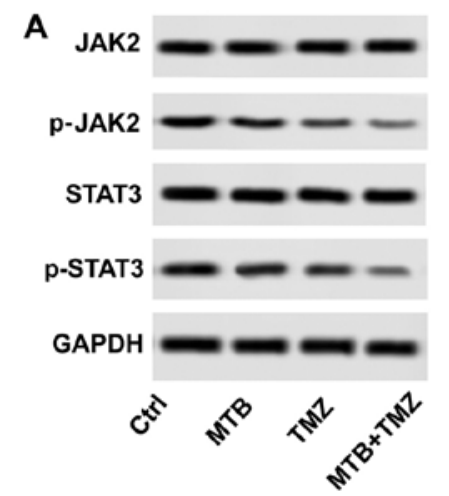

B

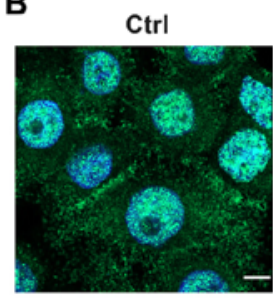

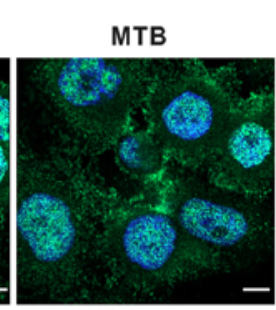
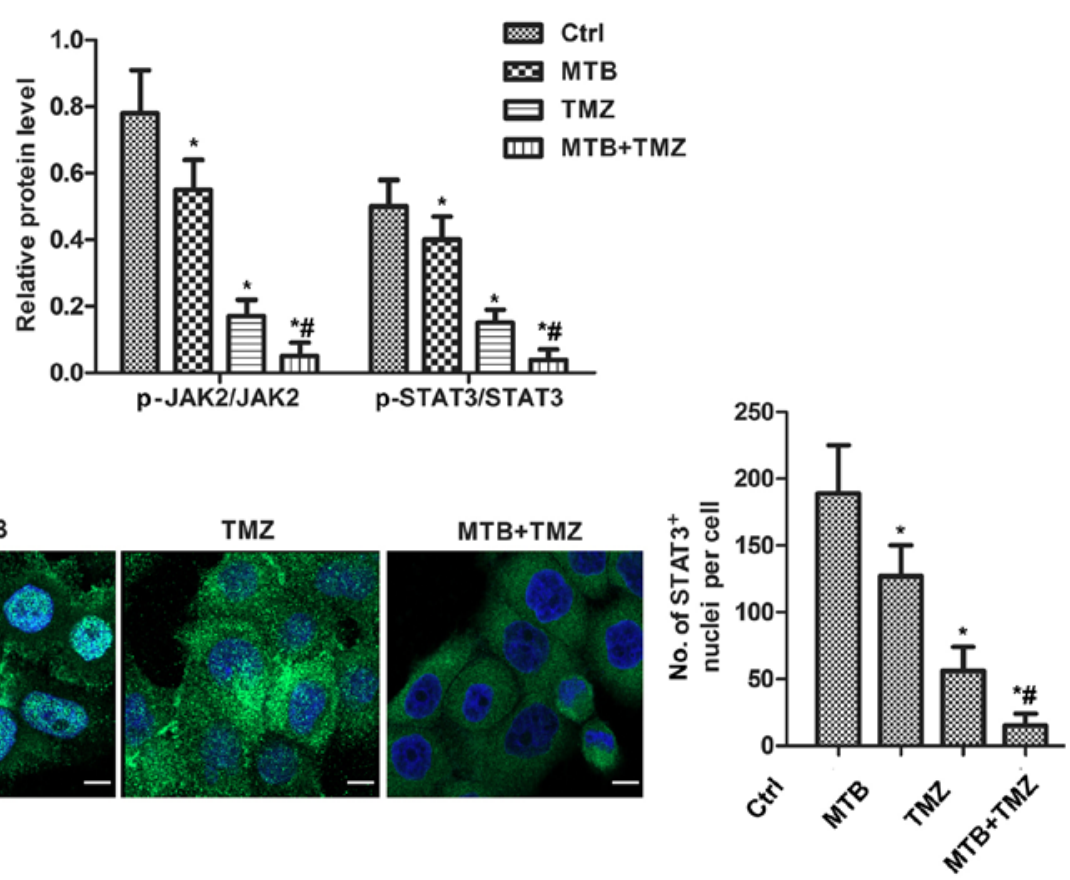

Figure 4. Treatment with the combination of MTB and TMZ inhibits the JAK2/STAT3 pathway in GBM cells in vitro. (A) Relative protein levels of JAK2, STAT3, p-JAK2 and p-STAT3 were detected by western blot analysis. GAPDH was used as loading control. The quantification of relative protein expression was performed by ImageJ. (B) Localization of STAT3 was visualized with fluorescence microscopy after immunofluorescence staining with STAT3 antibody (green). Cells were stained with DAPI for visualization of the cell nuclei (blue). The number of STAT3-positive staining nuclei were counted. "P $<0.05$, compared with Ctrl; " $\mathrm{P}<0.05$, compared with TMZ. Data are presented as the mean \pm SEM for 3 replicates; scale bar, $20 \mu \mathrm{m}$. MTB, momelotinib; TMZ, temozolomide; GBM, glioblastoma; STAT3, signal transducers and activators of transcription; p-STAT3, Tyr705-phosphorylated STAT3; JAK2, Janus kinase 2; p-JAK2, phosphorylated Janus kinase 2; Ctrl, control.

As shown in Fig. 4A, western blot analysis showed that the combination of MTB and TMZ decreased the phosphorylation levels of JAK2 and STAT3 compared to that following treatment with TMZ or MTB used alone at the same dose $(\mathrm{P}<0.05)$. In particular, MTB was observed to suppress STAT3 nuclear translocation. As shown in Fig. 4B, the nuclear level of p-STAT3 was high in the untreated U251 cells; whereas, the nuclear level of p-STAT3 was significantly reduced by MTB and TMZ. In addition, the nuclear level of p-STAT3 in the MTB + TMZ group was significantly decreased compared to that following TMZ or MTB used alone at the same dose $(\mathrm{P}<0.05)$.

$M T B$ enhances the growth-inhibiting effect and apoptosis-promoting of TMZ in U251 orthotopic xenograft models. We employed U-251MG orthotopic xenograft models to confirm the results of the combination treatment of $\mathrm{MTB}+\mathrm{TMZ}$ in vivo. MTB at $50 \mathrm{mg} / \mathrm{kg} / \mathrm{day}$ (body weight) (19) and TMZ at $20 \mathrm{mg} / \mathrm{kg} /$ day (MTB + TMZ) were administered by oral gavage. At day 30 after tumor cell implantation, mice were sacrificed for tumor weight analysis, immunohistochemistry, TUNEL and western blot assay. As shown in Fig. 5A, the combination treatment with $\mathrm{MTB}+\mathrm{TMZ}$ significantly decreased xenograft tumor weight compared to that following TMZ or MTB used alone at the same dose $(\mathrm{P}<0.05)$. As shown in Fig. 5B, the combination treatment with MTB and TMZ significantly increased the survival rate of the mice $(\mathrm{P}<0.05)$. In addition, the results of the TUNEL assay combined with immunohistochemistry demonstrated that the combination treatment with MTB and TMZ significantly promoted the apoptosis of the xenograft tumor cells (Fig. 5C and D; $\mathrm{P}<0.05$ ); immunohistochemistry of cleaved caspase- 3 confirmed an elevated apoptosis rate (Fig.5D). Meanwhile, the low expression of Ki-67 indicated suppression of proliferation following treatment with the combination of MTB and TMZ (Fig. 5D). Similarly, the combination of MTB and TMZ enhanced the autophagy-promoting effect in the xenograft tumors (Fig. 6A). Both TMZ and MTB decreased the phosphorylation of JAK2 and STAT3, while the combination of MTB and TMZ almost totally inhibited p-JAK2/p-STAT3 (Fig. 6B). The in vivo results were consistent with the in vitro findings.

\section{Discussion}

Temozolomide (TMZ) has been applied for the clinic treatment of gliomas as a first-line drug. However, TMZ resistance has become the most threatening challenge to glioblastoma multiforme (GBM) treatment. Therefore, it is vital to enhance the chemosensitivity of GBM to TMZ, which may contribute to prolong the survival time of these patients. In the present study, we demonstrated that $2.5 \mu \mathrm{M} / 50 \mathrm{mg} / \mathrm{kg}$ momelotinib (MTB) enhanced the chemosensitivity of GBM to TMZ both in vitro and in vivo.

The role of autophagy in GBM cells in response to metabolic and therapeutic stress seems to be different, including prosurvival and prodeath (22). Moreover, accumulating evidence has revealed a positive correlation between chemoresistance and autophagy reduction in $\operatorname{GBM}$ cells $(23,24)$. Therefore, autophagy induction may be a promising strategy to enhance the chemosensitivity of GBM to TMZ and improve 
A
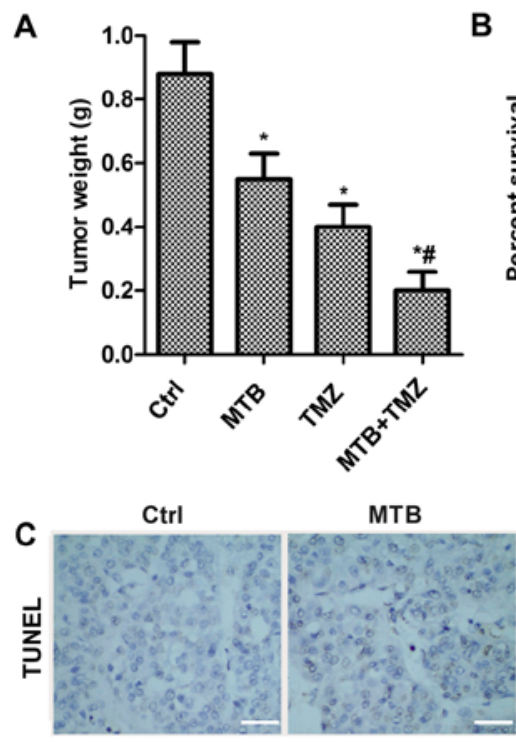

D
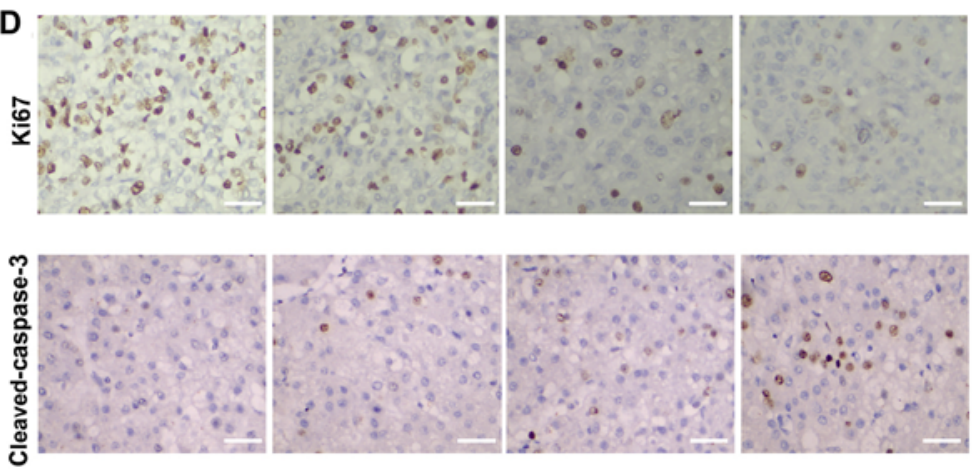

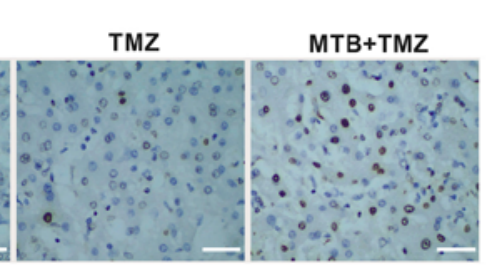

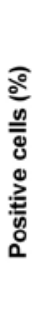

B

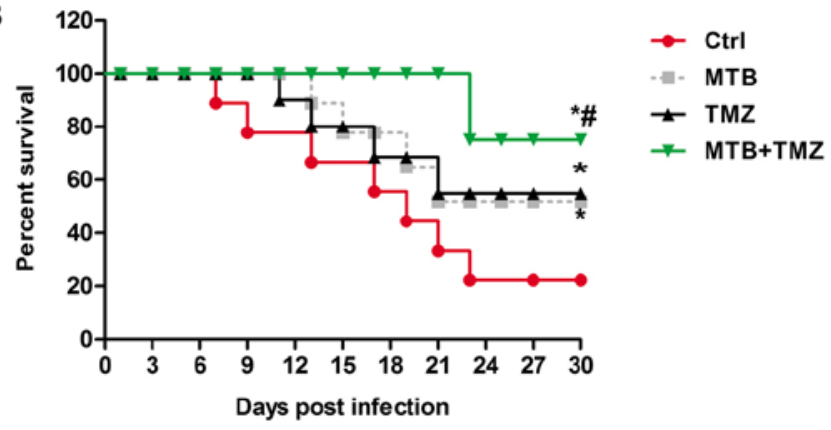

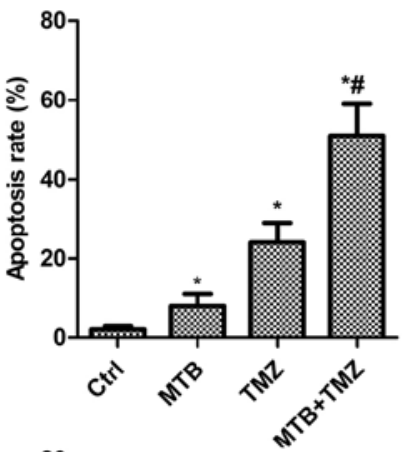

Ctrl

$\mathbf{\infty}$ мтв

曰

四 MTB+TMZ

Figure 5. MTB enhances the growth-inhibiting effect of TMZ in vivo. (A) Nude mice bearing subcutaneous xenograft GBM tumors were treated with Ctrl, MTB $(50 \mathrm{mg} / \mathrm{kg}$ of body weight), TMZ (20 mg/kg/day body weight) or MTB + TMZ. The weight of the tumors $(\mathrm{g})$ was determined at day 30 after mice were sacrificed. (B) The survival of mice was determined every 3 days for 30 days. (C) TUNEL assay was used to determine the apoptosis in the MTB, TMZ and combination treatment tumors compared with the NC (Ctrl)-treated tumors (magnification, $\mathrm{x} 400$ ); scale bar, $50 \mu \mathrm{m}$. (D) Immunohistochemical analysis of expression of Ki-67, cleaved-caspase-3 in the MTB, TMZ and combination treatment tumors compared with the NC (Ctrl)-treated tumors (magnification, $\mathrm{x} 400$ ); scale bar, $50 \mu \mathrm{m}$. Positive cells (brown) were counted. ${ }^{*} \mathrm{P}<0.05$, compared with $\mathrm{Ctrl} ;{ }^{*} \mathrm{P}<0.05$, compared with TMZ. The results are presented as the mean of 3 independent experiments. MTB, momelotinib; TMZ, temozolomide; GBM, glioblastoma; TUNEL, terminal deoxynucleotidyl transferase-mediated dUTP-biotin nick end labeling; Ctrl, control; caspase-3, cleaved-caspase-3.

the effectiveness of chemotherapy. For example, Bak et al reported that vitamin D enhanced autophagy in TMZ-based GBM chemotherapy (25). TMZ has been confirmed to induce autophagy in both glioma cells and surgical specimens $(26,27)$. Recently, another study demonstrated that $100 \mu \mathrm{M}$ TMZ induced autophagy AMPK-ULK1 pathways (28). MTB at $2.0 \mu \mathrm{M}$ was reported to induce autophagy in a human renal cell carcinoma (RCC) cell line by inhibiting mTOR complex 1 (29). In agreement with the previous results, our data found induction of autophagy with TMZ at a concentration of $100 \mu \mathrm{M}$ in vitro. In particular, $2.5 \mu \mathrm{M}$ MTB enhanced the autophagy of U251 cells induced by TMZ in vitro. Preclinical analysis has exhibited that MTB was well tolerated when administered to mice orally at doses up to $50 \mathrm{mg} / \mathrm{kg}$ of body weight, without obvious toxicity (30). In our in vivo study, $50 \mathrm{mg} / \mathrm{kg}$ MTB enhanced the autophagy induced by TMZ in nude mouse tumors. Thus, MTB enhanced the chemosensitivity of GBM to TMZ by potentiating TMZ-induced autophagy.

It has been well established that apoptosis is one of the promising therapeutic strategies for cancer treatment $(11,31)$.
Recently, research has demonstrated that apoptosis and the potentiating effects of chemotherapy response in GBM are positively related with STAT3 pathway inactivation $(13,32,33)$. Previous studies have revealed that Stat 3 activation was fully suppressed following the addition of MTB $(19,34,35)$. Importantly, MTB was reported to synergize with dasatinib to inhibit proliferation and induce apoptosis in RCC cell lines and suppress tumor growth in mouse xenograft models (19). In addition, MTB was reported to inhibit proliferation and induce apoptosis in myeloma cells via IL-6/JAK/STAT pathway inactivation (35). Research has indicated that apoptosis induction may lead to cancer cell impairment along with chemosensitivity enhancement (36-38). In the present study, it was found that MTB reduced the chemoresistance of GBM cells to TMZ by potentiating TMZ-induced apoptosis. The results were further supported by the finding that MTB + TMZ-treated xenografts had a significant decrease in tumor weight compared with that following TMZ alone treatment. Therefore, MTB may not only decease TMZ resistance in vitro, but also confine cancer growth in vivo. 

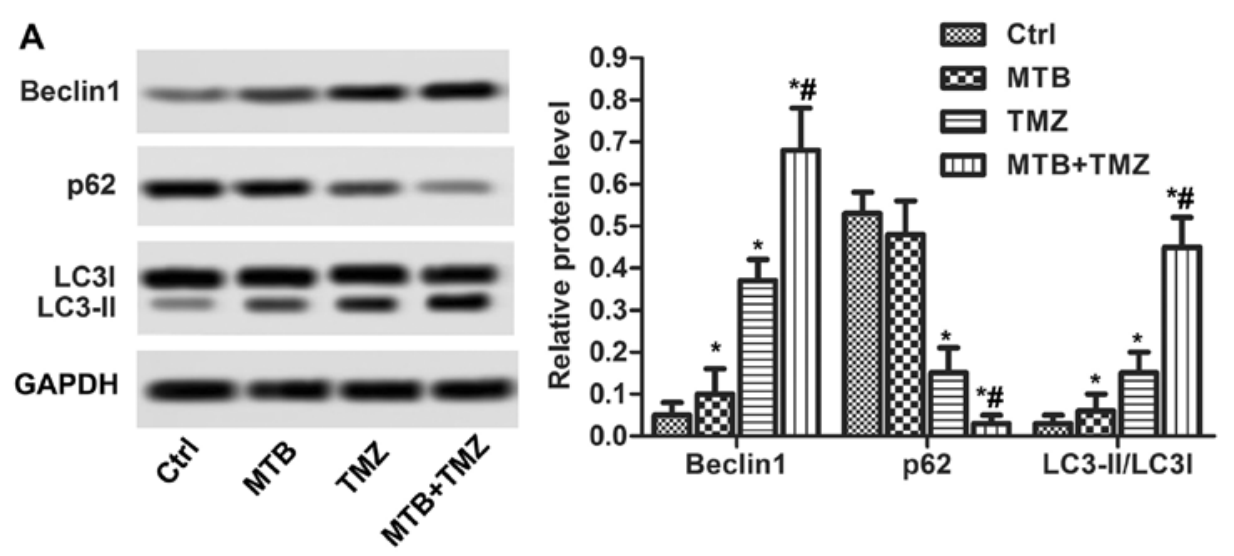

B
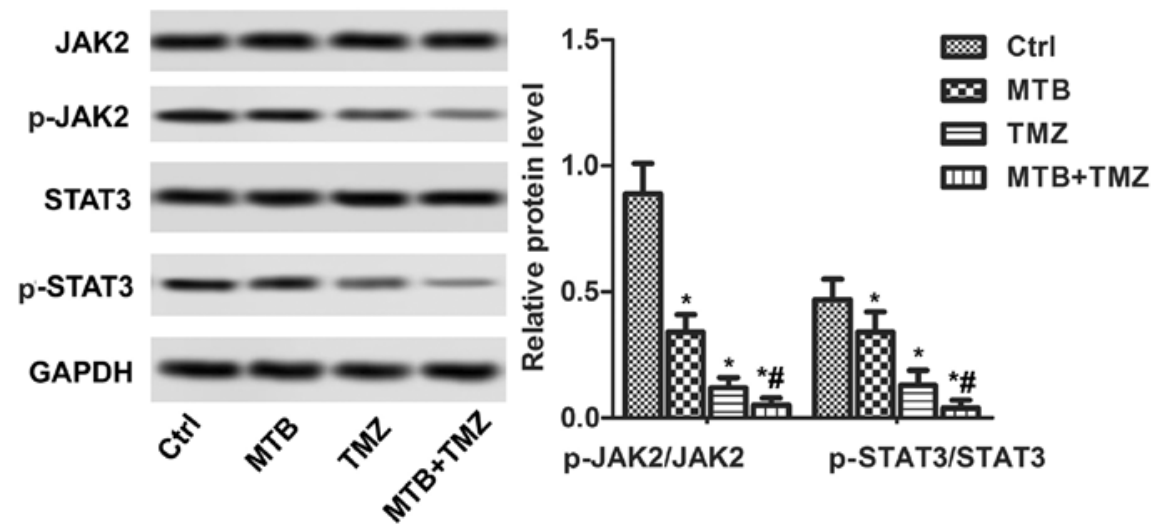

Figure 6. Treatment with the combination of MTB and TMZ inhibits the JAK2/STAT3 pathway in vivo. Nude mice (n=60) were treated with NC (Ctrl), MTB $(50 \mathrm{mg} / \mathrm{kg}$ body weight), TMZ ( $2 \mathrm{mg} / \mathrm{kg}$ body weight) or MTB + TMZ combination. At day 30, mice were sacrificed for immunohistochemistry, TUNEL and western blot assay. (A) Relative protein levels of Beclin1, LC3 I/II and p62 were detected by western blot analysis. GAPDH was used as loading control. The quantification of relative protein expression was performed by ImageJ. (B) Relative protein levels of JAK2, STAT3, p-JAK2 and p-STAT3 were detected by western blot analysis. GAPDH was used as a loading control. The quantification of relative protein expression was performed by ImageJ. ${ }^{*} \mathrm{P}<0.05$, compared with Ctrl; ${ }^{\text {P }}<0.05$, compared with TMZ. The results were presented as the mean of 3 independent experiments. MTB, momelotinib; TMZ, temozolomide; GBM, glioblastoma; STAT3, signal transducers and activators of transcription; p-STAT3, Tyr705-phosphorylated STAT3; JAK2, Janus kinase 2; p-JAK2, phosphorylated janus kinase 2; LC3, microtubule-associated protein 1 light chain 3; Ctrl, control.

JAK2/STAT3 pathway has been reported to play an important role in regulating cell growth, autophagy and apoptosis in various types of cancers, including breast cancer, esophageal carcinoma, colon cancer, osteosarcoma and ovarian cancer (39-43). In particular, STAT3 can be transiently activated by specific growth factors and cytokines in normal cells, while STAT3 remains constitutively active in certain populations of tumor cells, including breast, ovarian and prostate $(44,45)$. Notably, there exists a positive association between STAT3 constitutive activation and tumor grade in gliomas (9). Moreover, activation of STAT3 in cancer cells contributed to upregulation of multidrug resistance gene thus resulting in concomitant chemoresistance (34). It is well studied that the JAK2/STAT3 signaling pathway may be activated thus inducing tumor cell apoptosis (43) and autophagy (11). Our present study showed that MTB inhibited the phosphorylation of JAK2 and STAT3 in U251 cells, suggesting that MTB suppresses cell growth and chemoresistance through inactivation of the JAK2/STAT3 pathway. Collectively, it was demonstrated that MTB reduced the resistance of GBM to TMZ via induction of apoptosis induction and an increase in autophagy. Mechanistically, our findings indicate that MTB inactivates JAK2/STAT3 signaling thus triggering apoptosis and autophagy. As Bcl-2 and Bcl-xL are the downstream targets of STAT3, it is reasonable that MTB enhances the chemosensitivity of GBM via the MTB/JAK2/STAT3/Bcl2 axis.

In conclusion, MTB has anti-GBM potential both in GBM cells and U251 xenograft mouse models, and MTB enhances the antitumor effect of TMZ and chemosensitivity of GBM to TMZ via apoptosis and autophagy induction. The underlying mechanism may be attributed to the MTB/JAK2/STAT3/Bcl2 axis. Our data indicated that combination treatment with MTB and TMZ could provide a more effective therapeutic approach for GBM. In particularly, we found that MTB reduced the MGMT protein level. Due to the limited funding, we did not perform methylation detection. MTB may affect MGMT promoter methylation and this will be clarified in future research.

\section{Acknowledgements}

Not applicable.

\section{Funding}

The present study was supported by the National Natural Science Foundation of China (grant no. 81672481). 


\section{Availability of data and materials}

The datasets used during the present study are available from the corresponding author upon reasonable request.

\section{Authors' contributions}

$\mathrm{YXu}$ and $\mathrm{YXi}$ conceived and designed the study. TL, AL and $\mathrm{YXu}$ performed the experiments. TL and $\mathrm{YXi}$ wrote the paper. YXu and YXi reviewed and edited the manuscript. All authors read and approved the manuscript and agree to be accountable for all aspects of the research in ensuring that the accuracy or integrity of any part of the work are appropriately investigated and resolved.

\section{Ethics approval and consent to participate}

All animal protocols were approved by the Beijing Tiantan Hospital and Capital Medical University laws governing animal care.

\section{Patient consent for publication}

Not applicable.

\section{Competing interests}

The authors state that they have no competing interests.

\section{References}

1. Lathia JD, Mack SC, Mulkearns-Hubert EE, Valentim CL and Rich JN: Cancer stem cells in glioblastoma. Genes Dev 29: 1203-1217, 2015

2. Mahaley MS Jr, Mettlin C, Natarajan N, Laws ER Jr and Peace BB: National survey of patterns of care for brain-tumor patients. J Neurosurg 71: 826-836, 1989.

3. Athanassiou H, Synodinou M, Maragoudakis E, Paraskevaidis M, Verigos C, Misailidou D, Antonadou D, Saris G, Beroukas K and Karageorgis P: Randomized phase II study of temozolomide and radiotherapy compared with radiotherapy alone in newly diagnosed glioblastoma multiforme. J Clin Oncol 23: 2372-2377, 2005.

4. Johnson DR and O'Neill BP: Glioblastoma survival in the United States before and during the temozolomide era. J Neurooncol 107: 359-364, 2012.

5. Ostermann S, Csajka C, Buclin T, Leyvraz S, Lejeune F, Decosterd LA and Stupp R: Plasma and cerebrospinal fluid population pharmacokinetics of temozolomide in malignant glioma patients. Clin Cancer Res 10: 3728-3736, 2004.

6. Stupp R, Dietrich PY, Ostermann Kraljevic S, Pica A, Maillard I, Maeder P, Meuli R, Janzer R, Pizzolato G, Miralbell R, et al: Promising survival for patients with newly diagnosed glioblastoma multiforme treated with concomitant radiation plus temozolomide followed by adjuvant temozolomide. J Clin Oncol 20: 1375-1382, 2002.

7. Mukthavaram R, Ouyang X, Saklecha R, Jiang P, Nomura N, Pingle SC, Guo F, Makale M and Kesari S: Effect of the JAK2/STAT3 inhibitor SAR317461 on human glioblastoma tumorspheres. J Transl Med 13: 269, 2015.

8. Darnell JE Jr, Kerr IM and Stark GR: Jak-STAT pathways and transcriptional activation in response to IFNs and other extracellular signaling proteins. Science 264: 1415-1421, 1994.

9. Atkinson GP, Nozell SE and Benveniste ET: NF-kappaB and STAT3 signaling in glioma: Targets for future therapies. Expert Rev Neurother 10: 575-586, 2010.

10. Harada D, Takigawa $\mathrm{N}$ and Kiura K: The role of STAT3 in non-small cell lung cancer. Cancers 6: 708-722, 2014.
11. Song Y, Kong L, Sun B, Gao L, Chu P, Ahsan A, Qaed E, Lin Y, Peng J, Ma X, et al: Induction of autophagy by an oleanolic acid derivative, SZC017, promotes ROS-dependent apoptosis through Akt and JAK2/STAT3 signaling pathway in human lung cancer cells. Cell Biol Int 41: 1367-1378, 2017.

12. Zheng Q, Han L, Dong Y, Tian J, Huang W, Liu Z, Jia X, Jiang T, Zhang J, Li X, et al: JAK2/STAT3 targeted therapy suppresses tumor invasion via disruption of the EGFRvIII/JAK2/STAT3 axis and associated focal adhesion in EGFRvIII-expressing glioblastoma. Neurooncol 16: 1229-1243, 2014.

13. Stechishin OD, Luchman HA, Ruan Y, Blough MD, Nguyen SA, Kelly JJ, Cairncross JG and Weiss S: On-target JAK2/STAT3 inhibition slows disease progression in orthotopic xenografts of human glioblastoma brain tumor stem cells. Neuro Oncol 15: 198-207, 2013.

14. Höfener M, Pachl F, Kuster B and Sewald N: Inhibitor-based affinity probes for the investigation of JAK signaling pathways. Proteomics 15: 3066-3074, 2015.

15. Iurlo A and Cattaneo D: Treatment of myelofibrosis: Old and new strategies. Clin Med Insights Blood Disord 10: 1179545x17695233, 2017.

16. Pardanani A, Abdelrahman RA, Finke C, Lasho TT, Begna KH, Al-Kali A, Hogan WJ, Litzow MR, Hanson CA, Ketterling RP, et al: Genetic determinants of response and survival in momelotinib-treated patients with myelofibrosis. Leukemia 29: 741-744, 2015.

17. Yang S, Imamura Y, Jenkins RW, Cañadas I, Kitajima S, Aref A, Brannon A, Oki E, Castoreno A, Zhu Z, et al: Autophagy inhibition dysregulates TBK1 signaling and promotes pancreatic inflammation. Cancer Immunol Res 4: 520-530, 2016.

18. Chan E, Luwor R, Burns C, Kannourakis G, Findlay JK and Ahmed N: Momelotinib decreased cancer stem cell associated tumor burden and prolonged disease-free remission period in a mouse model of human ovarian cancer. Oncotarget 9: 16599-16618, 2018.

19. Lue HW, Cole B, Rao SA, Podolak J, Van Gaest A, King C, Eide CA, Wilmot B, Xue C, Spellman PT, et al: Src and STAT3 inhibitors synergize to promote tumor inhibition in renal cell carcinoma. Oncotarget 6: 44675-44687, 2015.

20. Hu Y, Dong XZ, Liu X, Liu P and Chen YB: Enhanced antitumor activity of cetuximab in combination with the Jak inhibitor CYT387 against non-small-cell lung cancer with various genotypes. Mol Pharm 13: 689-697, 2016.

21. Li J, Cai J, Zhao S, Yao K, Sun Y, Li Y, Chen L, Li R, Zhai X, Zhang J, et al: GANT61, a GLI inhibitor, sensitizes glioma cells to the temozolomide treatment. J Exp Clin Cancer Res 35: 184, 2016.

22. Li H, Chen L, Li JJ, Zhou Q, Huang A, Liu WW, Wang K, Gao L, Qi ST and Lu YT: $m i R-519 a$ enhances chemosensitivity and promotes autophagy in glioblastoma by targeting STAT3/Bcl2 signaling pathway. J Hematol Oncol 11: 70, 2018.

23. Shi F, Guo H, Zhang R, Liu H, Wu L, Wu Q, Liu J, Liu T and Zhang Q: The PI3K inhibitor GDC-0941 enhances radiosensitization and reduces chemoresistance to temozolomide in GBM cell lines. Neuroscience 346: 298-308, 2017.

24. Fu J, Liu ZG, Liu XM, Chen FR, Shi HL, Pangjesse CS, Ng HK and Chen ZP: Glioblastoma stem cells resistant to temozolomide-induced autophagy. Chin Med J 122: 1255-1259, 2009.

25. Bak DH, Kang SH, Choi DR, Gil MN, Yu KS, Jeong JH, Lee NS, Lee JH, Jeong YG, Kim DK, et al: Autophagy enhancement contributes to the synergistic effect of vitamin D in temozolomide-based glioblastoma chemotherapy. Exp Ther Med 11: 2153-2162, 2016

26. Natsumeda M, Aoki H, Miyahara H, Yajima N, Uzuka T, Toyoshima Y, Kakita A, Takahashi $\mathrm{H}$ and Fujii Y: Induction of autophagy in temozolomide treated malignant gliomas. Neuropathology 31: 486-493, 2011.

27. Lin CJ, Lee CC, Shih YL, Lin CH, Wang SH, Chen TH and Shih CM: Inhibition of mitochondria- and endoplasmic reticulum stress-mediated autophagy augments temozolomide-induced apoptosis in glioma cells. PLoS One 7: e38706, 2012.

28. Zou Y, Wang Q, Li B, Xie B and Wang W: Temozolomide induces autophagy via ATMAMPKULK1 pathways in glioma. Mol Med Rep 10: 411-416, 2014

29. Lue HW, Podolak J, Kolahi K, Cheng L, Rao S, Garg D, Xue CH, Rantala JK, Tyner JW, Thornburg KL, et al: Metabolic reprogramming ensures cancer cell survival despite oncogenic signaling blockade. Genes Dev 31: 2067-2084, 2017. 
30. Tyner JW, Bumm TG, Deininger J, Wood L, Aichberger KJ Loriaux MM, Druker BJ, Burns CJ, Fantino E and Deininger MW: CYT387, a novel JAK2 inhibitor, induces hematologic responses and normalizes inflammatory cytokines in murine myeloproliferative neoplasms. Blood 115: 5232-5240, 2010.

31. Cotter TG: Apoptosis and cancer: The genesis of a research field. Nat Rev Cancer 9: 501-507, 2009.

32. Wu J, Feng X, Zhang B, Li J, Xu X, Liu J, Wang X, Wang J and Tong X: Blocking the bFGF/STAT3 interaction through specific signaling pathways induces apoptosis in glioblastoma cells. J Neurooncol 120: 33-41, 2014.

33. Sai K, Wang S, Balasubramaniyan V, Conrad C, Lang FF, Aldape K, Szymanski S, Fokt I, Dasgupta A, Madden T, et al: Induction of cell-cycle arrest and apoptosis in glioblastoma stem-like cells by WP1193, a novel small molecule inhibitor of the JAK2/STAT3 pathway. J Neurooncol 107: 487-501, 2012.

34. Abubaker K, Luwor RB, Zhu H, McNally O, Quinn MA, Burns CJ, Thompson EW, Findlay JK and Ahmed N: Inhibition of the JAK2/STAT3 pathway in ovarian cancer results in the loss of cancer stem cell-like characteristics and a reduced tumor burden. BMC Cancer 14: 317, 2014.

35. Monaghan KA, Khong T, Burns CJ and Spencer A: The novel JAK inhibitor CYT387 suppresses multiple signalling pathways, prevents proliferation and induces apoptosis in phenotypically diverse myeloma cells. Leukemia 25: 1891-1899, 2011.

36. Beaujouin M, Baghdiguian S, Glondu-Lassis M, Berchem G and Liaudet-Coopman E: Overexpression of both catalytically active and -inactive cathepsin D by cancer cells enhances apoptosis-dependent chemo-sensitivity. Oncogene 25: 1967-1973, 2006.

37. Sun T, Jia Y and Xiao D: Interference of STAT 5b expression enhances the chemo-sensitivity of gastric cancer cells to gefitinib by promoting mitochondrial pathway-mediated cell apoptosis. Oncol Rep 34: 227-234, 2015.
38. Chen Y, Li R, Pan M, Shi Z, Yan W, Liu N, You Y, Zhang J and Wang X: MiR-181b modulates chemosensitivity of glioblastoma multiforme cells to temozolomide by targeting the epidermal growth factor receptor. J Neurooncol 133: 477-485, 2017.

39. Kim MS, Lee WS, Jeong J, Kim SJ and Jin W: Induction of metastatic potential by TrkB via activation of IL6/JAK2/STAT3 and PI3K/AKT signaling in breast cancer. Oncotarget 6: 40158-40171, 2015.

40. Sun KX, Xia HW and Xia RL: Anticancer effect of salidroside on colon cancer through inhibiting JAK2/STAT3 signaling pathway. Int J Clin Exp Pathol 8: 615-621, 2015.

41. Abubaker K, Luwor RB, Escalona R, McNally O, Quinn MA, Thompson EW, Findlay JK and Ahmed N: Targeted disruption of the JAK2/STAT3 pathway in combination with systemic administration of paclitaxel inhibits the priming of ovarian cancer stem cells leading to a reduced tumor burden. Front Oncol 4: 75, 2014

42. Liu JR, Wu WJ, Liu SX, Zuo LF, Wang Y, Yang JZ and Nan YM: Nimesulide inhibits the growth of human esophageal carcinoma cells by inactivating the JAK2/STAT3 pathway. Pathol Res Pract 211: 426-434, 2015.

43. Park KR, Yun HM, Quang TH, Oh H, Lee DS, Auh QS and Kim EC: 4-Methoxydalbergione suppresses growth and induces apoptosis in human osteosarcoma cells in vitro and in vivo xenograft model through down-regulation of the JAK2/STAT3 pathway. Oncotarget 7: 6960-6971, 2016.

44. Walker SR, Xiang M and Frank DA: Distinct roles of STAT3 and STAT5 in the pathogenesis and targeted therapy of breast cancer. Mol Cell Endocrinol 382: 616-621, 2014.

45. Liu C, Zhu Y, Lou W, Cui Y, Evans CP and Gao AC: Inhibition of constitutively active Stat 3 reverses enzalutamide resistance in LNCaP derivative prostate cancer cells. Prostate 74: 201-209, 2014. 